\title{
Tongue pressure resistance training for swallowing impairment post-stroke: study protocol for a randomized crossover clinical trial
}

Sana Smaoui ( $\sim$ sana.smaoui@uhn.ca )

Toronto Rehabilitation Institute https://orcid.org/0000-0001-6103-7585

Denyse Richardson

Toronto Rehabilitation Institute

Melanie Peladeau-Pigeon

Toronto Rehabilitation Institute

Catriona Steele

Toronto Rehabilitation Institute

Study protocol

Keywords: tongue pressure intervention, swallowing parameters in patients, dysphagia post-stroke

Posted Date: September 26th, 2019

DOI: https://doi.org/10.21203/rs.2.15195/v1

License: (c) (i) This work is licensed under a Creative Commons Attribution 4.0 International License. Read Full License 


\section{Abstract}

Introduction For patients who have suffered a stroke, tongue strength may be decreased compared to healthy individuals. Patients with dysphagia following stroke are more likely to experience aspiration pneumonia, which has been associated with higher mortality rates and medical complications. Research on strengthening the tongue in the stroke population has shown positive effects of a tongue resistance training protocol. Research also suggests that swallow safety, or protection of the airway, may be improved as a result of such interventions, however the mechanism of improvement remains poorly understood, particularly whether improvement is attributed to the intervention alone, or to spontaneous recovery. This study aims to determine what aspects of the swallowing mechanism (response time, movement, etc. of different structures) are directly impacted in order to provide guidance to clinicians using such treatments.

Methods and analysis A multi-site, assessor-blinded, randomized crossover trial was designed to determine the effects of a 4-week course of tongue pressure intervention supplemented with home practice in participants who experience dysphagia (PAS of 3 or greater) at baseline. An instrumental evaluation of swallowing (videofluoroscopic swallowing study) is utilized at baseline to determine whether swallowing safety is impaired. Additionally, a requirement for reduced tongue pressures $(<40$ kilopascals) on all three trials was added in order to target patients who would benefit from this training. Participants are randomized into a delayed treatment group or an immediate treatment group to control for the effects of spontaneous recovery. All participants receive the 4 week treatment consisting of 2 weekly sessions with a speech-language pathologist and complete daily homework plans. Videofluoroscopic swallowing studies are also performed pre and post intervention to quantify the degree of change attributed to the intervention. The study launched in May 2019 and will enroll a maximum of 40 participants.

Discussion This study will explore the effects of tongue pressure intervention on swallowing parameters in patients presenting with dysphagia post-stroke. Describing how the intervention effects specific swallowing mechanisms will provide guidance to clinicians using such treatments with this population. Trial registration number ClinicalTrials.gov identifier NCT03969095, published May 31 2019; https://clinicaltrials.gov/ct2/show/NCT03969095?term=NCT03969095\&rank=1

\section{Background And Rationale}

Swallowing dysfunction (dysphagia) following stroke results from damage to sensory and motor neuron pathways, impairing muscular function and impacting swallow timeliness leading to reduced airway protection. Patients with dysphagia experience impaired swallowing safety (entry of food or liquid into the airway, i.e., aspiration) and/or impaired swallowing efficiency (food or liquid remaining in the oropharyngeal cavities after the swallow, i.e., residue). Patients with dysphagia following stroke are more likely to experience aspiration pneumonia, which has been associated with higher mortality rates $[1,2]$. 
The tongue plays a key role in swallowing, containing the bolus within the oral cavity and generating the primary driving forces required to carry it through the pharynx. Consequently, impairments in tongue function can affect both swallowing safety and efficiency. For patients who have experienced a stroke, lingual strength is decreased compared to healthy participants [3]. Tongue pressure resistance training has been utilized as an intervention in this population, and has been shown to positively influence tongue strength [4-8]. However, it remains unclear whether increases in tongue pressure amplitude directly impact swallowing physiology, safety, and/or efficiency for patients who complete these interventions [9]. In particular, the impact of tongue pressure resistance training on swallow timeliness (swallow onset timing, time-to-laryngeal vestibule closure, speed of hyolaryngeal movement) remains unknown. In this study, we propose a randomized cross-over trial of tongue pressure resistance training in people with tongue weakness and impaired swallowing safety post-stroke. Our goal is to understand the physiological changes that occur following tongue pressure resistance training and their relationship to swallowing safety and efficiency.

Here we outline the protocol for a multi-site, assessor-blinded, randomized crossover trial to determine the effects of a 4-week course of tongue pressure intervention supplemented with home practice. Participants are randomly allocated into either an immediate or delayed intervention group. During the intervention, participants receive intensive, task-specific practice including one-on-one therapy with a Speech-Language Pathologist (S-LP) using a biofeedback instrument. Participants in both groups receive the same training program with identical session durations, intensity, frequency and repetitions. The main difference between groups is time-point at which the intervention is administered (see figure 1 below); this design allows for the exploration of spontaneous swallowing recovery in the delayed intervention group.

\section{Insert Figure 1 about here}

The primary objective of this study is to determine the results of this treatment program on swallowing safety outcomes as captured on instrumental videofluoroscopic swallow study (VFSS) assessment using the Penetration-Aspiration Scale (PAS) [10]. The secondary objectives include exploring effects of the intervention on (1) pixel based measures of post-swallow residue [11] and number of swallows per bolus to characterize swallowing efficiency (2) International Dysphagia Diet Standardisation Initiative Functional Diet Scale (IDDSI-FDS) to represent the degree of diet texture modification [12] (3) other swallowing physiology parameters (timing and kinematics) [13]. We hypothesize that the intervention will result in improved swallowing safety and positively impact timing and kinematics of swallow events. For our secondary objectives, we hypothesize that participation in the study will result in a reduction in vallecular residue and an increase in IDDSI-FDS scores.

\section{Methods/design}

\section{Study Design}


A multi-center, assessor-blinded randomized crossover clinical trial (ClinicalTrials.gov identifier NCT03969095, Registered 31 May 2019; https://clinicaltrials.gov/ct2/show/NCT03969095?term = NCT03969095\&rank = 1) titled Tongue Pressure Resistance Training for Swallowing Impairment PostStroke (TPRT-SIPS) is being conducted over 3 years at the University Health Network-Toronto Rehabilitation Institute-University Centre in Toronto, Ontario, Canada and Northwestern MedicineMarianjoy Hospital in Wheaton, Illinois, USA. These sites each have stroke programs that provide inpatient and outpatient services.

Informed consent is obtained from each participant prior to enrollment in the study. Once obtained, participants are assigned a unique study participant number used on all documentation. Three trials of maximum isometric tongue pressures are collected at the anterior location from each participant. Eligibility to proceed to the next part of the study is achieved if they display decreased tongue pressures measured using the lowa Oral Performance Instrument (IOPI) as compared to healthy normal individuals ( $<40$ kilopascals) on all three trials. A VFSS is then completed to confirm impaired swallowing safety and therefore, eligibility for study inclusion.

Participants are enrolled and receive a computer generated randomization number if a PAS score of 3 or above is observed by a study member on any of the 8 swallowing trials in the baseline VFSS assessment. All participants complete a baseline VFSS, 4-week course of tongue-pressure resistance training followed by a VFSS, and a 4-week no-treatment/detraining phase followed by a VFSS regardless of allocation, see Table 1. 


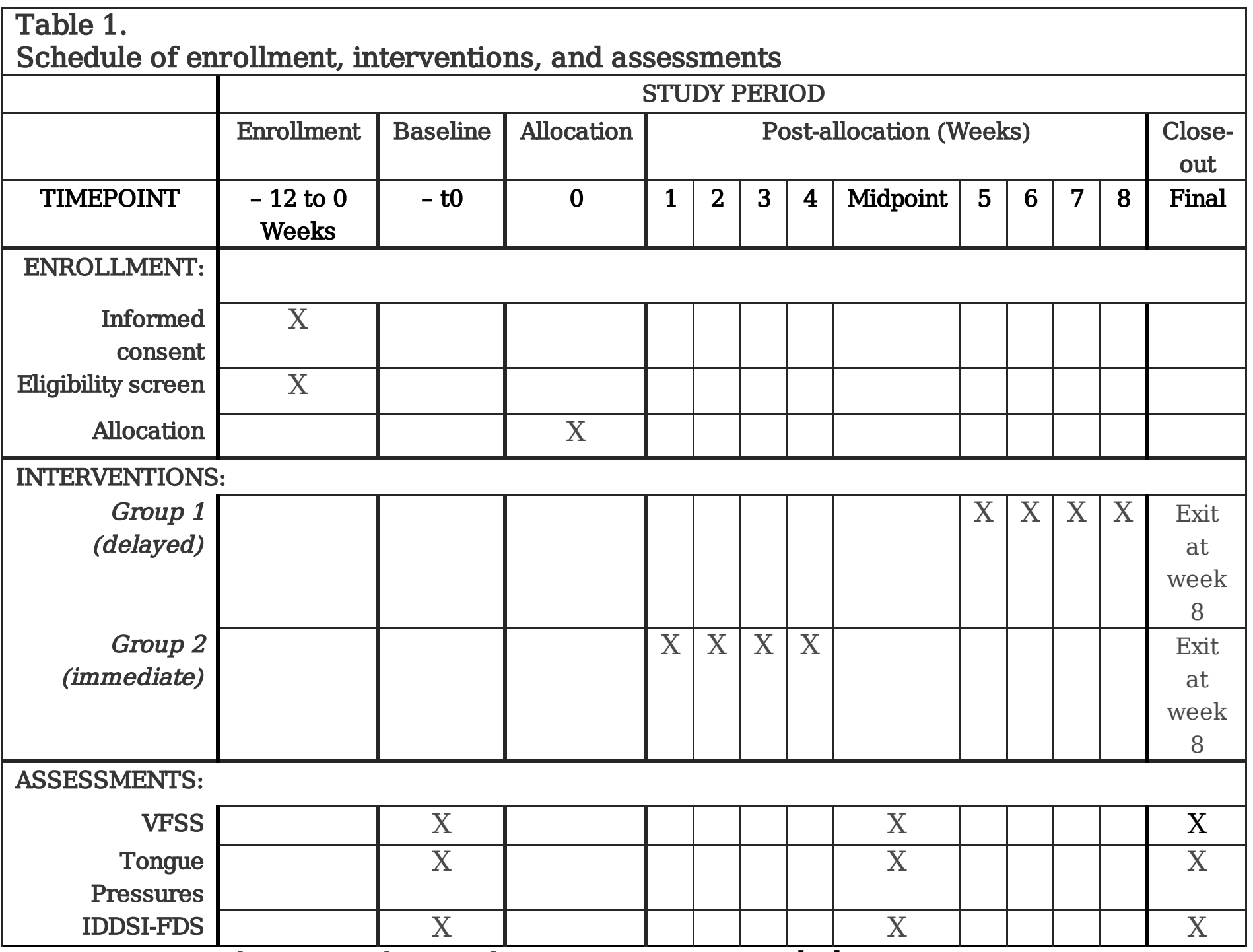

Participant timeline according to the SPIRIT Statement [14] VFSS Videofluoroscopy, IDDSI-FDS: International Dysphagia Diet Standardisation Initiative.

We propose the enrollment of a study sample of approximately 40 participants. Participants will be allocated either to a delayed treatment group involving a 4-week waiting period prior to beginning the 4week training or to an immediate treatment group (followed by a 4-week post-treatment detraining phase). The allocation sequence for randomization numbers is stored in password protected files at the primary study site where the lab manager is the only individual with access to this concealed sequence until interventions are to be assigned. Once safety concerns are identified on baseline assessment, study members reach out to the lab manager to assign the participant and allocation to either group in the trial. The sample size calculation for this study was based on 2-sided chi-square testing for association between treatment group (Immediate and Delayed) and swallowing safety (Safe vs. Unsafe). A study with a power calculation of 0.8 , and standard deviation of proportion of unsafe swallows set at 0.5 will require a sample size of 32 participants ( $n=16$ per group) to test the association at a 0.05 significance level. The power calculation was carried out using Study Size 3.0. We will enroll a total of 40 participants $(n=20$ 
per group) to account for participant attrition. The use of a crossover design will allow for the evaluation of outcomes at different stroke time points and attaining the required level of statistical power, along with the quantification of swallow recovery with and without treatment exposure. Each participant will serve as their own control, while allowing for an exploration of treatment effects and their interaction with neuronal recovery periods for statistical analyses.

\section{Participants}

All participants are approached by a member of their circle of care who introduces the study. Screening for inclusion is completed through review of both the paper chart and the electronic medical record. Participants then meet with a member of the research team who reviews study procedures and obtains informed consent from each participant. Inclusion and exclusion criterial are detailed in Table 2.

\section{Table 2. \\ Trial Inclusion and Exclusion Criteria}

\begin{tabular}{|c|c|}
\hline Inclusion Criteria & Exclusion Criteria \\
\hline $1 . \geq 18$ years old & 1. History of head and neck cancer \\
\hline 2. Stroke type: ischemic stroke & 2. Radical neck dissection (e.g. anterior cervical \\
\hline (first-ever) & surgery fusions) or neck/ oropharyngeal surgery \\
\hline 3. Stroke region: Supra-tentorial & (not excluded - tonsillectomy, adenoidectomy, \\
\hline point: Within 3 months of & heostomy) \\
\hline stroke & 3. Past medical history of any neurological disease \\
\hline 5. Diagnosis confirmed via & other than stroke (e.g. MS, PD, ALS, TBI) \\
\hline neuroimaging techniques & 4. Oral apraxia, impairing the participant's ability \\
\hline magnetic resonance imaging) & 5. Cognitive or receptive communication \\
\hline $\begin{array}{l}\text { 6. Decreased tongue pressures on } \\
\text { IOPI }\end{array}$ & $\begin{array}{l}\text { difficulties that preclude the participant's ability } \\
\text { to follow study instructions }\end{array}$ \\
\hline $\begin{array}{l}\text { 7. Pharyngeal dysphagia (safety } \\
\text { concern operationalized as a score } \\
\text { of } 3 \text { or more on the penetration- } \\
\text { aspiration scale) }\end{array}$ & $\begin{array}{l}\text { 4. Allergies to barium, potato starch, corn starch, } \\
\text { xanthan gum, milk products, or latex }\end{array}$ \\
\hline
\end{tabular}

\section{Standardized VFSS protocol}

Each participant will complete instrumental videofluoroscopic swallowing studies at three time-points in the study based on their group allocation. These studies will utilize a pulse rate of 30 pulses per second to generate 30 images per second, which will be recorded using a properly synchronized recording system (either the KayPentax Digital Swallow Workstation or the TIMS DICOM System) at 30 frames per second. The VFSS protocol involves up to four natural cup sips of Level 0 thin liquid stimuli and up to four natural cup sips of Level 2 mildly thick liquid stimuli as defined by the International Dysphagia Diet Standardisation Initiative (www.iddsi.org). All liquids are mixed with barium sulfate in a $20 \%$ weight-tovolume concentration using either Bracco E-Z-Paque ${ }^{\circledR}$ or Varibar $^{\circledR}$ Thin Liquid powdered barium. The 
mildly thick liquids are prepared using a xanthan gum based thickening agent (Nestlé Resource ${ }^{\circledR}$ ThickenUp ${ }^{\circledR}$ Clear). Participants are provided with cups containing 40 milliliters of liquid for each sip, and cups are weighed pre and post administration to calculate the total volume of liquids swallowed per sip. Of the four mildly thick liquid trials, two are completed with the IOPI bulb positioned anteriorly (directly behind the upper incisors) in order to collect tongue pressures during swallowing. The VFSS protocol is outlined in Figure 2.

\section{Insert Figure 2 about here}

In order to ensure that participants are not exposed to harm through participation in the study, a number of discontinuation rules will be employed during each VFSS:

- If aspiration of liquid (Penetration-Aspiration Scale of 6 or more) is observed during the study for a certain consistency, that consistency or method of administration (e.g., with IOPI in mouth) will no longer be offered to the participant.

- When three instances of aspiration have been observed in total, the VFSS exam will be terminated.

- If the vallecular or pyriform sinus spaces are overflowing with residue with potential danger of entry into the airway, participants will be cued to swallow a second time to clear the residue. If this is ineffective, that method of administration will no longer be offered to the participant.

- If the duration of radiation exposure exceeds 3.5 minutes in total, the VFSS will be concluded.

\section{Training Program:}

The training protocol consists of a total of 8 intervention sessions completed twice per week over the span of four weeks. Study participants are asked from completion the following exercises during the trial: effortful swallows, Shaker exercise, Mendelsohn maneuver, Masako Maneuver, jaw opening against resistance, and chin tuck against resistance as they may interfere with trial results. Each session will be supervised by an S-LP and require a maximum of an hour in length. The program is designed to utilize a combination of strengthening tasks alongside swallowing tasks using biofeedback in order to optimize generalization to real-life swallowing conditions. Sessions completed with the S-LPs will be comprised of the following:

- 20 repetitions of maximum isometric presses (MIPs) emphasizing rapid generation of pressure ("rapid rise") at the anterior palate bulb location. A mark will be made by the S-LP on the connecting tube of the bulb at the level of the incisors at the beginning of the session to ensure accurate and consistent placement of the bulb during practice.

- 15 regular saliva swallows with the IOPI bulb in place. The mark on the connecting tube will be aligned with the upper incisors. A mandatory rest period of 30 seconds will be utilized per saliva swallow. 
- 15 effortful saliva swallows with the IOPI bulb in place. The mark on the connecting tube will be aligned with the upper incisors. A mandatory rest period of 30 seconds will be utilized per saliva swallow.

- 10 generalization tasks with the IOPI in place along with a mildly thick liquid sip. The mark on the connecting tube will be aligned with the upper incisors, then one natural cup sip of mildly thick liquid will be provided for each swallow.

Additionally a home practice component using IOPI bulbs with a target of 30 additional repetitions daily of the MIP and the Saliva Swallow tasks is included. Participants will be provided with a daily homework log to document practice, and will review this log weekly with their S-LP.

A participant will be withdrawn from the study if they miss a total of 3 consecutive intervention sessions under the direct supervision of an SL-P, or develop a new health concern.

\section{Outcome measures:}

Tongue pressures using the IOPI device using different tasks repeated three times each will be collected at each encounter during the study. Maximum isometric tongue pressures will be collected at the (1) anterior location where the tongue bulb will be positioned behind the upper incisors and the participant will be instructed to squeeze the bulb hard; and (2) posterior location lining the tongue bulb with the molar teeth with similar instruction to squeeze the bulb hard. Regular effort saliva swallow pressures will also be collected, where the tongue bulb is again lined up behind the upper incisors and participants are instructed to swallow their saliva with normal effort, allowing at least 30 seconds between trials.

International Dysphagia Diet Standardisation Initiative Functional Diet Scale (IDDSI-FDS) scores will be collected at each encounter to monitor the degree of diet texture modification used and the time-points at which any changes in diet texture occur relative to the treatment program.

Videofluoroscopic swallowing measures will be collected at baseline, post 4-week waiting/detraining period, and post 4-week treatment for each group using a standardized videofluoroscopy protocol. VFSS recordings are de-identified, spliced into separate clips for each bolus, and arranged in a randomized order for duplicate rating. Rating is completed at the Toronto Rehabilitation Institute site by two S-LPS at the Swallowing Rehabilitation Research Laboratory (SRRL) trained using the ASPEKT method [13] and blinded to participant and VFSS time point for the following parameters:

- Penetration-Aspiration Scale (PAS)

- Pixel-based measures of post-swallow residue

- Swallow Reaction Time

- Time-to-Laryngeal Vestibule Closure

- Laryngeal Vestibule Closure Duration 
- Hyoid burst kinematics (Hyoid velocity and peak position)

- Pharyngeal Area at Rest

- Pharyngeal Area at Maximal Pharyngeal Constriction

The primary outcome of interest is swallowing safety as measured using the penetration-aspiration scale.

\section{Confidentiality}

All participant data will be coded using an alphanumeric study code. The master key linking the study code to patient identity will be maintained at the University Health Network-Toronto Rehabilitation Institute-University Centre, and by the lead investigators at Northwestern Medicine-Marianjoy Hospital. Personnel involved in the study (recruitment, consent, and completing the intervention) will have direct access to the study related documents. VFSS studies will be de-identified and stored electronically on a secure, password-protected, encrypted research drive at the University Health Network-Toronto Rehabilitation Institute-University Centre. Any data collected on paper will be inputted into electronic documents and shredded.

VFSS data and scanned copies of paper study forms from the Northwestern Medicine-Marianjoy Hospital site will be transferred to the University Health Network-Toronto Rehabilitation InstituteUniversity Centre using OneDrive. As patient VFSS studies are considered personal health information and are protected under the Personal Health Information Protection Act (PHIPA), they will be archived at the end of the study and stored at University Health Network-Toronto Rehabilitation Institute-University Centre for a period of 10 years after which they will be disposed of in a secure manner.

\section{Statistical analysis}

Excel software will be used for data extraction and IBM SPSS Statistics will be used for statistical analysis. Swallowing safety and efficiency measures will be transformed to binary measures (e.g. safe/unsafe; efficient/inefficient) in order to facilitate comparisons. As we are dealing with categorical variables as our primary exposure and outcome variables, a binary logistic regression model will be used to explore the association, if any, between the treatment groups and the transformed swallow safety measures. Other possible variables that have been identified by the literature to possibly influence this association will be taken into account and included in the model (covariates: of time-since-stroke, timepoint; modifier: stroke severity; stroke location).

For continuous measures of swallowing physiology, parametric analyses, such as repeated measures ANOVAs, will be used to detect overall differences between related means across different conditions (bolus types) and time points (pre-post intervention).

\section{Discussion}


Although tongue pressure training is a therapeutic intervention used by S-LPs to treat swallowing impairment in the context of decreased tongue pressures, the current literature lacks evidence with regard to the efficacy of this intervention compared to spontaneous recovery. This protocol targets participants under the diagnostic category of stroke presenting with concurrent swallow safety concerns and decreased tongue pressures. Mechanistically, changes in tongue pressure may increase oral control of liquid boluses thus aiding in the generation of driving forces that influence swallowing physiology related to safety and/or efficiency. Gains in swallowing function following tongue pressure interventions cannot be solely attributed to the intervention itself if the period of spontaneous recovery is not accounted for. The use of a cross-over design has allowed us to control for spontaneous recovery, which needs to be accounted for in a patient population where some spontaneous recovery can occur[15]. This protocol actively targets tongue pressure training during swallowing tasks to promote generalization of the task, utilizing principles of neural recovery as a basis for its design. If found to be effective, this treatment program may inform best practice in dysphagia rehabilitation for this patient population.

\section{Declarations}

\section{Ethics approval and consent to participate}

This protocol was approved by the Research Ethics Board (REB) at University Health Network-Toronto Rehabilitation Institute-University Centre on April 2, 2019 and Institutional Review Board (IRB) at Northwestern Medicine-Marianjoy Hospital on April 25 2019. Consent is obtained in writing from all participants. Any modifications to the protocol which may impact on the study will undergo a formal amendment to the protocol and will be communicated to all relevant parties including: investigators, $\mathrm{REB} / \mathrm{IRB}$, trial participants, and trial registries.

\section{Trial Status}

The study has been recruiting since May 2019, and it is estimated that the recruitment will end in May 2021. The current protocol version is dated 17 June 2019.

\section{Consent for publication}

Not applicable

\section{Availability of data and materials}

Only the coordinating site (University Health Network-Toronto Rehabilitation Institute-University Centre) will have access to the full final trial data set. The investigators from each participating site will have 
access to their own site's data sets. The trial protocol and copies of trial documentation may be available from the corresponding author on reasonable request.

\section{Competing interests}

The liquids for the study will be prepared with Bracco EZPaque Barium powder and with a thickening agent manufactured by Nestlé (ThickenUp Clear ${ }^{\mathrm{T}}{ }^{\mathrm{M}}$ ). These products have been chosen because they are readily available and approved for clinical use in Canada. CMS has past and current research relationships with Bracco Canada and with Nestlé Health Science. She also serves in an advisory capacity to Nestlé Health Science on expert panels. Neither Bracco Canada nor Nestlé Health Science are involved as sponsors of this study and they will not have access to the study data. The products for this study will be purchased by the hospital. CMS will not receive any payments (either personally or to the lab) from Bracco Canada or Nestlé Health Science related to the use of these products in the study. DR, the co-investigator, does not have any conflicts of interest to declare. SS and MPP do not have any conflicts of interest to declare.

\section{Funding}

This trial is funded by a Heart and Stroke Foundation-Canadian Partnership for Stroke Recovery Catalyst Grant. Any decisions regarding study design, execution, analyses, interpretation of the data, or publication of results are not the responsibility of the funding source.

\section{Authors' contributions}

SS and CMS conceived the study. All authors (SS, DR, MPP, CMS) have contributed to clinical trial protocol design and have read and approved the final manuscript

\section{Acknowledgements}

Not applicable.

\section{Abbreviations}

VFSS: videofluoroscopic swallowing study

PAS: penetration-aspiration scale

IDDSI: international dysphagia diet standardisation initiative

IDDSI-FDS: international dysphagia diet standardisation initiative-functional diet scale 
IOPI: iowa oral performance instrument

MIPs: maximum isometric pressures.

\section{References}

1. Mann G, Hankey GJ, Cameron D (1999) Swallowing function after stroke: prognosis and prognostic factors at 6 months. Stroke 30:744-748

2. Martino R, Foley N, Bhogal S, Diamant N, Speechley M, Teasell R (2005) Dysphagia after stroke: incidence, diagnosis, and pulmonary complications. Stroke 36:2756-2763. doi:

10.1161/01.STR.0000190056.76543.eb

3. Hori K, Ono T, Iwata H, Nokubi T, Kumakura I (2005) Tongue pressure against hard palate during swallowing in post-stroke patients. Gerodontology 22:227-233. doi: 10.1111/j.17412358.2005.00089.x

4. Cho Y-S, Oh D-H, Paik Y-R, Lee J-H, Park J-S (2017) Effects of bedside self-exercise on oropharyngeal swallowing function in stroke patients with dysphagia: a pilot study. J Phys Ther Sci 29:1815-1816. doi: 10.1589/jpts.29.1815

5. Kim HD, Choi JB, Yoo SJ, Chang MY, Lee SW, Park JS (2017) Tongue-to-palate resistance training improves tongue strength and oropharyngeal swallowing function in subacute stroke survivors with dysphagia. Journal of Oral Rehabilitation 44:59-64. doi: 10.1111/joor.12461

6. Park J-S, Kim H-J, Oh D-H (2015) Effect of tongue strength training using the lowa Oral Performance Instrument in stroke patients with dysphagia. J Phys Ther Sci 27:3631-3634. doi:

$10.1589 /$ jpts.27.3631

7. Robbins J, Kays SA, Gangnon RE, Hind JA, Hewitt AL, Gentry LR, Taylor AJ (2007) The Effects of Lingual Exercise in Stroke Patients With Dysphagia. Archives of Physical Medicine and Rehabilitation 88:150-158. doi: 10.1016/j.apmr.2006.11.002

8. Steele CM, Bayley MT, Peladeau-Pigeon M, Nagy A, Namasivayam AM, Stokely SL, Wolkin T (2016) A Randomized Trial Comparing Two Tongue-Pressure Resistance Training Protocols for Post-Stroke Dysphagia. Dysphagia 31:452-461. doi: 10.1007/s00455-016-9699-5

9. McKenna VS, Zhang B, Haines MB, Kelchner LN (2017) A Systematic Review of Isometric Lingual Strength-Training Programs in Adults With and Without Dysphagia. Am J Speech Lang Pathol 26:524-539. doi: 10.1044/2016_AJSLP-15-0051

10. Rosenbek JC, Robbins JA, Roecker EB, Coyle JL, Wood JL (1996) A penetration-aspiration scale. Dysphagia 11:93-98. doi: 10.1007/BF00417897

11. Pearson WG, Molfenter SM, Smith ZM, Steele CM (2013) Image-based Measurement of PostSwallow Residue: The Normalized Residue Ratio Scale. Dysphagia 28:167-177. doi: $10.1007 /$ s00455-012-9426-9

12. Steele CM, Namasivayam-MacDonald AM, Guida BT, Cichero JA, Duivestein J, Hanson B, Lam P, Riquelme LF (2018) Creation and Initial Validation of the International Dysphagia Diet 
Standardisation Initiative Functional Diet Scale. Archives of Physical Medicine and Rehabilitation 99:934-944. doi: 10.1016/j.apmr.2018.01.012

13. Steele CM, Peladeau-Pigeon M, Barbon CAE, Guida BT, Namasivayam-MacDonald AM, Nascimento WV, Smaoui S, Tapson MS, Valenzano TJ, Waito AA, Wolkin TS (2019) Reference Values for Healthy Swallowing Across the Range From Thin to Extremely Thick Liquids. J Speech Lang Hear Res 62:1338-1363. doi: 10.1044/2019_JSLHR-S-18-0448

14. Chan A-W, Tetzlaff JM, Gøtzsche PC, Altman DG, Mann H, Berlin JA, Dickersin K, Hróbjartsson A, Schulz KF, Parulekar WR, Krleža-Jerić K, Laupacis A, Moher D (2013) SPIRIT 2013 explanation and elaboration: guidance for protocols of clinical trials. BMJ 346:e7586. doi: 10.1136/bmj.e7586

15. Cramer SC (2008) Repairing the human brain after stroke: I. Mechanisms of spontaneous recovery. Annals of Neurology 63:272-287. doi: 10.1002/ana.21393

\section{Figures}




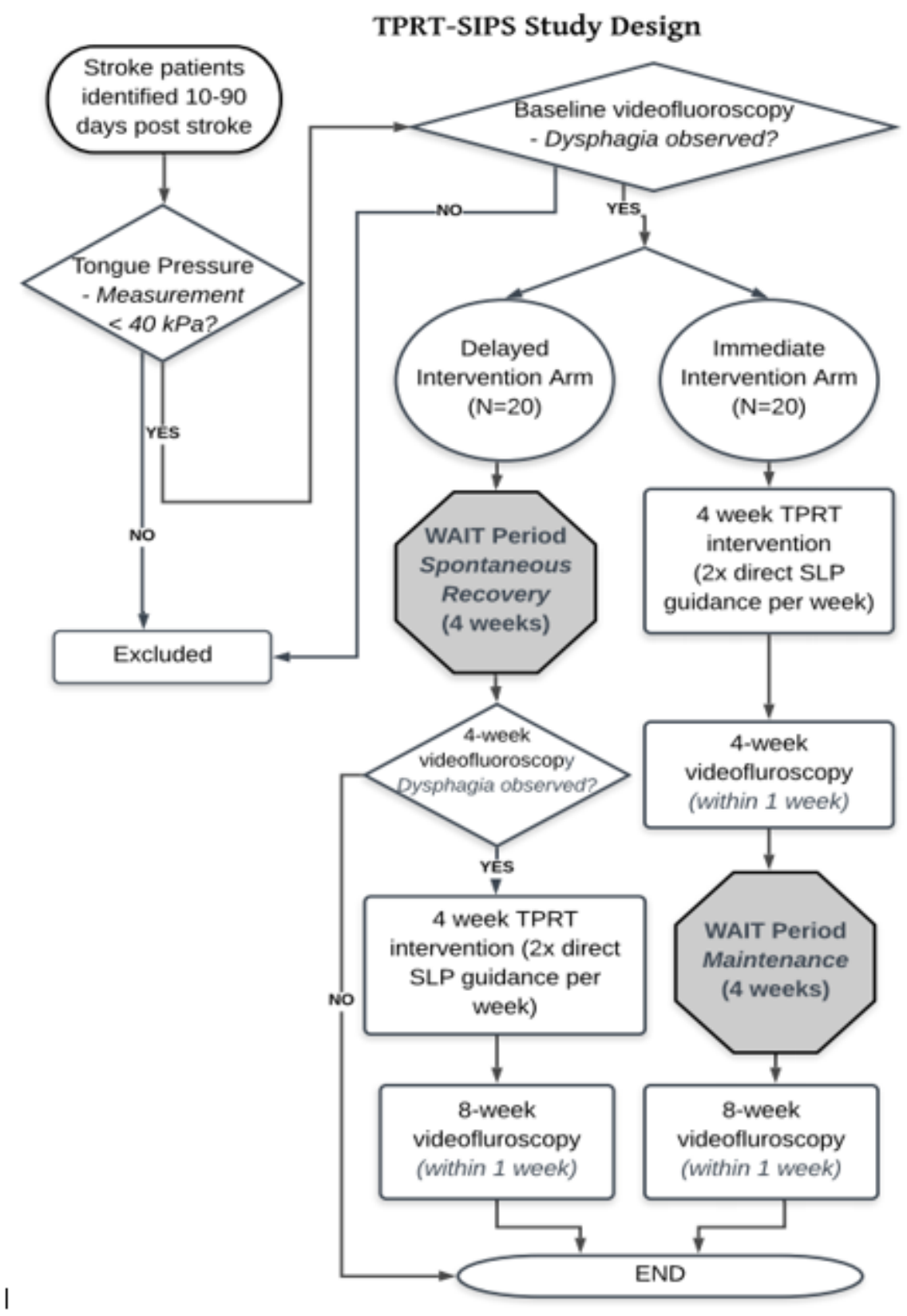

Figure 1

TPRT-SIPS study design 


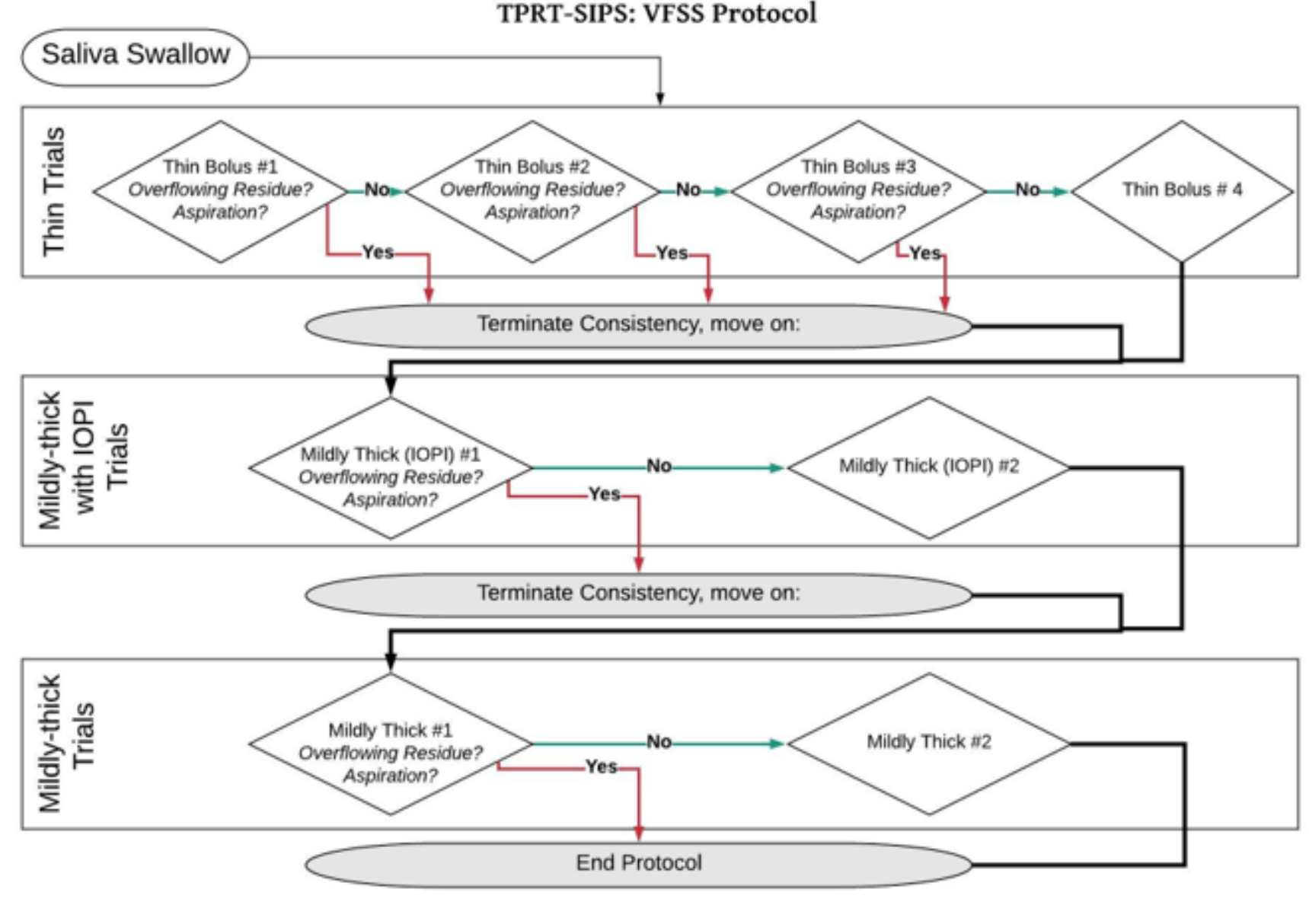

Figure 2

VFSS protocol

\section{Supplementary Files}

This is a list of supplementary files associated with this preprint. Click to download.

- SPIRITchecklist.pdf 